\title{
Risk Assessment of Injury to Palmar Cutaneous Branch of the Median Nerve Using High- Resolution Ultrasound
}

\author{
Young Ha Jeong, MD, Jun Ho Choi, MD, Hyuk Sung Choi, MD, Seok Kang, MD, PhD, \\ Seung Nam Yang, MD, PhD, Joon Shik Yoon, MD, PhD \\ Department of Physical Medicine and Rehabilitation, Korea University Guro Hospital, Seoul, Korea
}

Objective To evaluate the relationship between the palmar cutaneous branch of median nerve (PCBMN) and surrounding anatomical structures by using high-resolution ultrasound (HRUS) to assess the risk of PCBMN injury.

Methods The PCBMN course and the characteristics of bilateral distal forearms and wrists of 30 healthy volunteers were identified. The distance between PCBMN and other anatomical structures at three different levels along its course were measured using HRUS. Moreover, the depth of PCBMN from skin and its cross-sectional area (CSA) were measured.

Results HRUS showed the PCBMN in all subjects. PCBMN branched off from the radial aspect of the median nerve $(\mathrm{MN})$ at $4.69 \pm 0.89 \mathrm{~cm}$ proximal to the bistyloid line (BSL) and extended radially toward the flexor carpi radialis (FCR) tendon. PCBMN was within the ulnar edge of FCR tendon sheath, and became more superficial and perforated the antebrachial fascia between the FCR tendon laterally and the palmaris longus (PL) tendon medially. PCBMN was located at $4.08 \pm 0.72 \mathrm{~mm}$ on the ulnar aspect of the FCR tendon and $4.78 \pm 0.36 \mathrm{~mm}$ radially on the PL tendon at BSL. At the distal wrist crease level, the PCBMN was located at $5.68 \pm 0.58 \mathrm{~mm}$ on the ulnar side of the FCR tendon. The PCBMN depth from skin at BSL and its branching point was $1.92 \pm 0.41$ and $7.95 \pm 0.79 \mathrm{~mm}$, respectively. The PCBMN CSA was $0.26 \pm 0.15 \mathrm{~mm}^{2}$ at BSL.

Conclusion HRUS can be used to identify PCBMN and its relationship with other anatomical structures. Our data can be used to predict PCBMN location, and prevent complications associated with invasive procedures involving the wrist.

Keywords Palmar cutaneous branch, Median nerve, Ultrasound, Carpal tunnel syndrome Yoon (http://orcid.org/0000-0002-8985-279X).

(c) This is an open-access article distributed under the terms of the Creative Commons Attribution Non-Commercial License (http://creativecommons.org/ licenses/by-nc/4.0) which permits unrestricted noncommercial use, distribution, and reproduction in any medium, provided the original work is properly cited. Copyright () 2019 by Korean Academy of Rehabilitation Medicine 


\section{INTRODUCTION}

The palmar cutaneous branch of the median nerve (PCBMN) is the last collateral branch of the median nerve $(\mathrm{MN})$ in the wrist before entering the carpal tunnel [1]. The PCBMN arises from the distal forearm before the $\mathrm{MN}$ enters the carpal tunnel, and then runs along its own tunnel in the antebrachial fascia and traverses superficially to the flexor retinaculum $[2,3]$. This small branch of the MN provides sensory innervation to the thenar eminence and the proximal part of the palm. If the PCBMN is damaged due to trauma or entrapment in the distal forearm or wrist, severe sensory symptoms such as permanent hypoesthesia or paresthesia may occur in the area innervated by the PCBMN (so-called pillar pain) $[4,5]$.

Previous studies have shown that invasive procedures performed on the distal forearm or wrist, including volar plating of the distal radius fracture and carpal tunnel release are associated with the risk of iatrogenic damage to the PCBMN [6-8]. Carroll and Green [6] first reported that the paresthesia and neuroma of the PCBMN could be induced after carpal tunnel release. Louis et al. [7] reported that injury to the PCBMN is the most common complication of carpal tunnel surgery. The modified Henry approach, which is commonly performed during the operative treatment of distal radius fractures, requires incision through the flexor carpi radialis (FCR) tendon sheath to facilitate retraction of the FCR tendon towards the ulnar aspect. Given that the PCBMN runs almost parallel to the ulnar aspect of the FCR tendon, the modified Henry approach increases the risk of injury to this nerve during surgery [8]. Thus, identifying and understanding the anatomical course of the PCBMN are important for surgeons performing invasive procedures on the distal forearm or wrist.

Electrodiagnostic examination may be used to evaluate lesions of the PCBMN [9]. Imai reported that nerve conduction study for the PCBMN was highly sensitive (75\%) and specific (100\%) for diagnosing entrapment of the PCBMN preoperatively. However, this test is not always reliable because of the extensive overlap of the sensory distribution of the MN and the PCBMN [10].

High-resolution ultrasound (HRUS) is another option for evaluation of the PCBMN $[11,12]$. Given that HRUS facilitates excellent tissue differentiation for the examination of superficial structures, we hypothesize that the evaluation of PCBMN is possible over the entire course of the nerve. Although several cadaveric studies have focused on the anatomical relationship between the PCBMN and the surrounding structures $[2,13,14]$, the relationship between the PCBMN and the surrounding structures using HRUS has yet to be reported.

The aim of this study was to evaluate the course of PCBMN and its relationship with the surrounding anatomical structures by using HRUS, to assess the risk of injury to the PCBMN, and to suggest safe approaches with minimal risk of complications during invasive interventions involving the distal forearm or wrist.

\section{MATERIALS AND METHODS}

Thirty heathy adults (15 male and 15 female participants) volunteered for this study between March 1, 2018 and August 1, 2018. Bilateral wrists were examined in each subject (a total of 60 wrists). Participants manifested no neurological symptoms, clinical signs, or abnormal findings upon neurological examinations suggestive of neuropathy of the PCBMN or the MN. Participants with a history of forearm surgery or trauma and systemic diseases, such as diabetes mellitus, were excluded. This study was approved by the Institutional Review Board of the Korea University Guro Hospital (No. 2018GR0132), and all participants provided written informed consent before the study.

The wrists were scanned using HRUS (RS85; Samsung Medison, Seoul, Korea) interfaced with a 3-16 MHz linear array transducer (hockey stick probe). All sonographic studies were performed by the same physiatrist.

During the examination, the healthy volunteers were seated in front of the physiatrist and were asked to place their hands on the table with palms facing upwards. They were also asked to supinate their forearms fully and bend their fingers slightly for examination. The scanning technique relied on images obtained in the transverse plane between the MN and FCR tendon. To better identify the PCBMN, the examiner performed dynamic scanning by sweeping the probe slowly up and down over its course (Fig. 1). This method enabled the identification of PCBMN, which exhibited a continuous hypoechoic structure surrounded by soft tissue echoes and the point where this nerve penetrated the fascia. Moreover, to differentiate this small cutaneous nerve, a real-time com- 


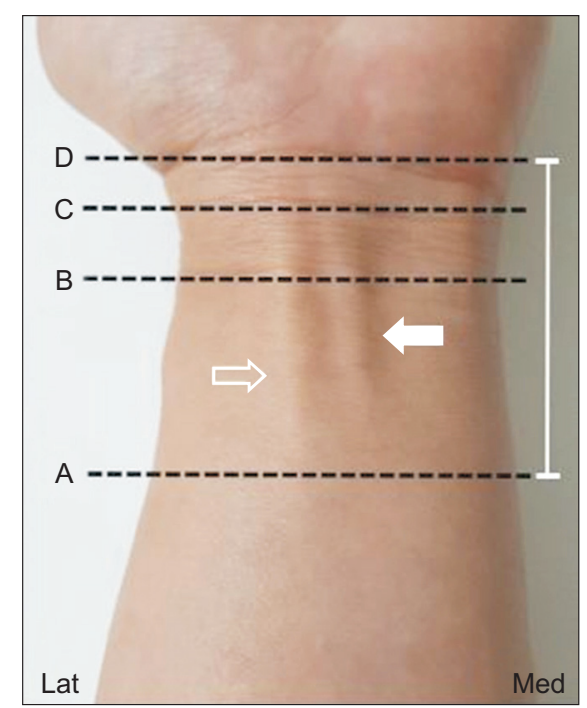

Fig. 1. The levels of sonographic scanning around the wrist to detect PCBMN: ' $\mathrm{A}$ ' is branching point of PCBMN from $\mathrm{MN}$, 'B' proximal wrist crease, ' $\mathrm{C}$ ' bistyloid line, and ' $\mathrm{D}$ ' distal wrist crease (open arrow indicates FCR tendon; solid arrow, PL tendon; and white arrow line, distance between DWC and branching point of the PCBMN from MN). PCBMN, palmar cutaneous branch of the median nerve; MN, median nerve; FCR, flexor carpi radialis; PL, palmaris longus; DWC, distal wrist crease. pressing and releasing method or Doppler ultrasonography was used.

After identifying the course of PCBMN, we measured the distance between PCBMN and other anatomical structures, including the palmaris longus tendon (PL), FCR tendon and MN at the bistyloid line (BSL) (Fig. 2C). We defined the BSL, which is the line between the lateral side of the styloid process of the radius and the medial side of the styloid process of the ulna as shown in a previous cadaveric study [2]. We also measured the distance between PCBMN from the FCR tendon and MN at the distal wrist crease (DWC) (Fig. 2D). The distance between the branching point of the PCBMN from MN and DWC was measured. The depths of PCBMN from skin at the BSL (Fig. 2C) and the branching point of PCBMN (Fig. $2 \mathrm{~A}$ ) were also measured. All measured distances in crosssectional sonogram were calculated using the caliper mode of the HRUS device. At the BSL, the cross-sectional area (CSA) of PCBMN was measured using the continuous trace mode of the HRUS device.

\section{RESULTS}

Demographic data are shown in Table 1. In our study,
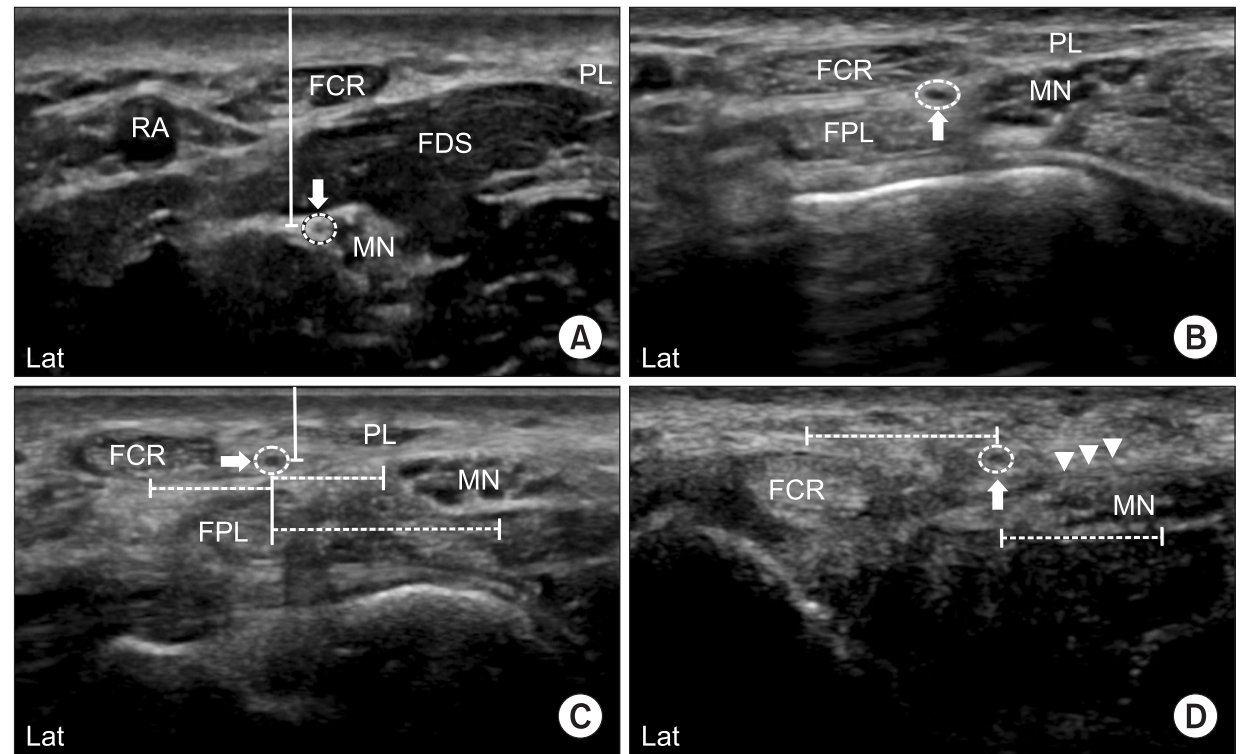

Fig. 2. Cross-sectional sonogram demonstrating distances between PCBMN and surrounding anatomic structures at different levels, as presented in Fig. 1. (A) Solid line indicates depth of PCBMN from skin, and solid arrow, PCBMN, (B) solid arrow, PCBMN, (C) dashed line, distances between PCBMN from the FCR tendon, PL tendon and MN; and solid arrow, PCBMN, and (D) dashed line, distances between PCBMN from the FCR tendon and MN; arrowhead, flexor retinaculum; solid arrow, PCBMN. PCBMN, palmar cutaneous branch of the median nerve; MN, median nerve; FCR, flexor carpi radialis; PL, palmaris longus. 
Table 1. Demographic data of the study participants

\begin{tabular}{lcc}
\hline & Men $(\mathbf{n}=15)$ & Women $(\mathbf{n}=15)$ \\
\hline Age $(\mathrm{yr})$ & $31.23 \pm 9.52$ & $30.58 \pm 11.78$ \\
\hline Height $(\mathrm{m})$ & $1.76 \pm 0.06$ & $1.61 \pm 0.04$ \\
Weight $(\mathrm{kg})$ & $73.23 \pm 8.68$ & $51.08 \pm 6.89$ \\
BMI $\left(\mathrm{kg} / \mathrm{m}^{2}\right)$ & $23.67 \pm 2.11$ & $20.15 \pm 2.32$ \\
\hline Forearm length $(\mathrm{cm})$ & $26.21 \pm 2.12$ & $25.86 \pm 1.42$ \\
\hline
\end{tabular}

Values are presented as mean \pm standard deviation. BMI, body mass index.

Table 2. Morphometric measurement of PCBMN using HRUS

\begin{tabular}{lcc}
\hline \multicolumn{1}{c}{ Measurement } & Average & $\begin{array}{c}\text { Standard } \\
\text { deviation }\end{array}$ \\
\hline CSA of PCBMN $\left(\mathrm{mm}^{2}\right)$ & 0.26 & 0.15 \\
Depth at BSL $(\mathrm{mm})$ & 1.92 & 0.41 \\
FCR to PCBMN at BSL (mm) & 4.08 & 0.72 \\
FCR to PCBMN at DWC (mm) & 5.68 & 0.58 \\
PL to PCBMN at BSL (mm) & 4.78 & 0.36 \\
MN to PCBMN at BSL $(\mathrm{mm})$ & 5.81 & 0.87 \\
MN to PCBMN at DWC $(\mathrm{mm})$ & 7.05 & 0.61 \\
Branching point from DWC $(\mathrm{cm})$ & 4.69 & 0.89 \\
Depth at the branching point $(\mathrm{mm})$ & 7.95 & 0.79 \\
\hline
\end{tabular}

PCBMN, palmar cutaneous branch of the median nerve; HRUS, high-resolution ultrasound; CSA, cross-sectional area; BSL, bistyloid line; DWC, distal wrist crease; FCR, flexor carpi radialis; PL, palmaris longus; $\mathrm{MN}$, median nerve.
HRUS was able to distinguish the PCBMN in all wrists (60 wrists). All the scanned morphometric data are shown in Table 2 and Fig. 3. At the branching point of PCBMN from the MN, the PCBMN was visible from the radial aspect of the MN in all cases. The PCBMN branched off from the radial aspect of the $\mathrm{MN}$ at $4.69 \pm 0.89 \mathrm{~cm}$ proximal from the DWC and extended radially toward the FCR tendon. The PCBMN continued its course alongside the MN until it approached the FCR tendon and ran within the ulnar edge of the sheath of the FCR tendon, without crossing the FCR tendon. The PCBMN became more superficial and perforated the antebrachial fascia of the distal forearm just proximal to the BSL, between the FCR tendon laterally and the MN medially. Then, this cutaneous nerve moved to the superficial aspect of the flexor retinaculum. At the BSL, the PCBMN was located at $4.08 \pm 0.72 \mathrm{~mm}$ on the ulnar aspect of the FCR tendon and at $4.78 \pm 0.36 \mathrm{~mm}$ on the radial aspect of the PL tendon. At the DWC, the PCBMN was located at $5.68 \pm 0.58 \mathrm{~mm}$ on the ulnar aspect of the FCR tendon, above the flexor retinaculum. The depth of the PCBMN from skin at the BSL was $1.92 \pm 0.41 \mathrm{~mm}$. The distances between PCMBN and $\mathrm{MN}$ at two different levels (BSL and DWC) were 5.81 \pm 0.87 and $7.05 \pm 0.61$, respectively. The depth of the PCBMN from skin at the branching point was $7.95 \pm 0.79 \mathrm{~mm}$. In the transverse scan, PCBMN appeared as a single, rounded and hypoechoic fascicle along its course, and its CSA was measured as $0.26 \pm 0.15 \mathrm{~mm}^{2}$ at the BSL.

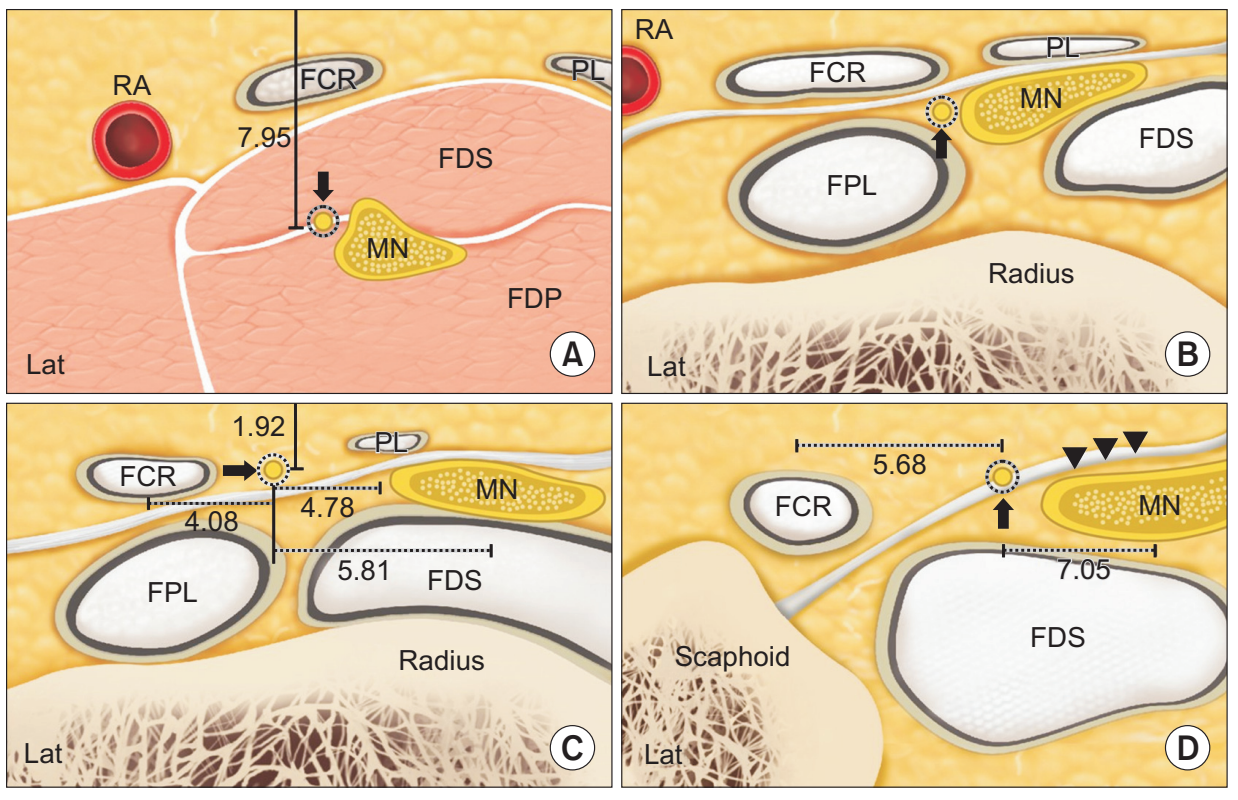

Fig. 3. Schematic diagram of cross-sectional sonogram showing measured distances $(\mathrm{mm})$ in Fig. 2. $\mathrm{RA}$, radial artery; $\mathrm{MN}$, median nerve; FCR, flexor carpi radialis; $\mathrm{PL}$, palmaris longus; FDS, flexor digitorum superficialis; FPL, flexor pollicis longus; Lat, lateral. 


\section{DISCUSSION}

In this study, we investigated the relationship between the PCBMN and the surrounding anatomical structures using HRUS to assess the risk of PCBMN injury. The results of this study confirmed that the PCBMN can be reliably visualized and its entire course can be evaluated using HRUS. Although our results are based on healthy volunteers and additional studies are required to clarify the role of HRUS, we think that HRUS may play a critical role in assessing the risk of injury to the PCBMN during operative or invasive procedures around the distal forearm or wrist, and in evaluating patients with suspected PCBMN neuropathies.

The PCBMN is the last collateral branch of the MN that provides sensation to the thenar eminence and the palmar skin of the hand [15]. The clinical value of the PC$\mathrm{MBN}$ is related to its vulnerability during various surgical procedures around the distal forearm and wrist, including carpal tunnel release for carpal tunnel syndrome, volar plating of the radius for distal radius fracture, tendon transfer of the PL, and resection of ventral carpal ganglion $[6,16,17]$. Damage to PCBMN is associated with chronic wrist pain, pillar pain, increased scar tenderness, and complex regional pain syndrome [4]. The incidence of complex regional pain syndrome due to smaller branches including PCMBN has been estimated in 3\% to $10 \%$ of cases after open reduction and volar plating of the distal radius [18].

Several cadaveric studies investigated the anatomy of the PCBMN, mainly designed to prevent injury to the PCBMN in palmar incisions for carpal tunnel release and volar plating for distal radius fracture, based on its anatomical course $[2,14]$. Chaynes et al. [2] reported that the PCBMN in all cases originated in the radial side of the $\mathrm{MN}$ at a mean distance of $44.5 \mathrm{~mm}$ proximal to the BSL. Conti Mica et al. [14] found the separation point at an average distance of $48.6 \mathrm{~mm}$ proximal from the DWC. In our study, the PCBMN originated in the MN, at an average distance of $46.9 \mathrm{~mm}$ proximal to the DWC. The branching point of the PCBMN, measured by using HRUS, was similar to the location described in previous anatomical studies using cadavers $[2,14]$.

McCann et al. [13] dissected 10 fresh cadavers and reported that PCBMN was located at a distance of $3.4 \mathrm{~mm}$ on average from the ulnar aspect of the FCR tendon in the watershed area of the distal radius. Our study showed that the PCBMN was located at an average distance of 4.08 $\mathrm{mm}$ from the ulnar aspect of the FCR tendon and at 4.78 $\mathrm{mm}$ from the radial aspect of the PL tendon at the BSL. In our study, at the DWC, the PCBMN was located at 5.68 $\mathrm{mm}$ on average from the ulnar aspect of the FCR tendon. Our results explain the risk of injury to the PCBMN associated with the carpal tunnel release, especially when an incorrect incision extends proximal to the wrist crease in the area between the PL tendon and the FCR tendon.

A previous cadaveric study reported that the PCBMN runs along the trunk of the MN for 10-25 $\mathrm{mm}$, and then moves radially toward the FCR tendon before perforating the antebrachial fascia, and becoming more superficial and perforating the antebrachial fascia between the FCR tendon laterally and the PL tendon medially [2]. The PCBMN runs within the FCR tendon sheath before perforating the antebrachial fascia, thereby increasing the risk of potential injury during surgeries, including the modified Henry's approach of volar plating, which requires incision through the FCR tendon and traction of FCR tendon to the ulnar side [14]. The overall course of the PCBMN, suggested by the cadaver dissection is supported by the results of our sonographic study.

We measured the depths of the PCBMN at two different levels (BSL and branch point from MN). The depth at which PCBMN is located and the branching point of the PCBMN from the MN can be confirmed with HRUS. Therefore, clinical practitioners including hand surgeons can understand the three-dimensional location and course of the PCBMN in each patient, and apply the technique in clinical practice before performing invasive procedures on the wrist.

Several previous studies have reported that the ulnar approach is the safest technique for carpal tunnel injection in patients with carpal tunnel syndrome $[19,20]$. In our data, the distance between the MN and PCBMN was an average of $5.81 \mathrm{~mm}$ at the BSL and $7.05 \mathrm{~mm}$ at the DWC. Considering the positional relationship between PCBMN and MN near the carpal tunnel based on ultrasound, our sonographic study revealed that the ulnar approach for carpal tunnel injection is superior to other techniques, especially the radial approach, for the prevention of PCBMN injury.

Sonographic quantification of the CSA of the nerve plays a critical role in the assessment of neuropathy for 
peripheral nerves [21]. In the present study, the CSA of the PCBMN has an average of $0.26 \mathrm{~mm}^{2}$ at the BSL. Based on ultrasound measurement, Tagliafico et al. [11] have suggested that the CSA of the PCBMN is $0.5-0.7 \mathrm{~mm}^{2}$ at the point where the MN crosses between the FCR tendon and the radial boundary of the flexor digitorum superficialis muscle. Although our measurement was performed at a somewhat anatomically distal site, our measurements are thought to be smaller than those of the previous study, probably due to factors such as race (in this study, Asian) or body mass index. Based on these results, HRUS examination of a patient suspected with impaired PCBMN may facilitate clinical application as HRUS can be used to detect changes in CSA.

Several limitations of this study should be considered. First, the small sample size might have influenced the results, especially in the detection of rare courses or absence of this nerve [22]. Although this study examined a greater number of wrists than the previous sonographic study, our data are limited to 30 healthy volunteers, and anomalous relationships are possible between these structures in less common anatomical configurations. Second, only a single physician performed the nerve measurements; therefore, a definitive statement about the diagnostic reliability of the PCBMN measurements may be limited. Third, we did not confirm the findings via anatomical dissection. To confirm the existence of small cutaneous nerves, the sonographic study must be accompanied with an anatomical study based on cadaver dissection.

In conclusion, HRUS can be used to identify the anatomical course and characteristics of the PCBMN. We recommend its use in clinical practice, such as during the pre-evaluation of carpal tunnel release, open reduction and internal fixation for distal radius fracture, and so on.

\section{CONFLICT OF INTEREST}

No potential conflict of interest relevant to this article was reported.

\section{AUTHOR CONTRIBUTION}

Conceptualization: Jeong YH, Yoon JS. Methodology: Jeong YH, Yoon JS. Formal analysis: Jeong YH. Funding acquisition: Yoon JS. Project administration: Choi JH,
Choi HS. Visualization: Jeong YH. Writing - original draft: Jeong YH. Writing - review and editing: Yang SN, Kang S, Yoon JS. Approval of final manuscript: all authors.

\section{REFERENCES}

1. Bezerra AJ, Carvalho VC, Nucci A. An anatomical study of the palmar cutaneous branch of the median nerve. Surg Radiol Anat 1986;8:183-8.

2. Chaynes P, Becue J, Vaysse P, Laude M. Relationships of the palmar cutaneous branch of the median nerve: a morphometric study. Surg Radiol Anat 2004;26:27580.

3. Rotman MB, Donovan JP. Practical anatomy of the carpal tunnel. Hand Clin 2002;18:219-30.

4. Kuhlmann N, Tubiana R, Lisfranc R. Contribution of anatomy to the understanding of carpal tunnel compression syndromes and sequelae of decompression operations. Rev Chir Orthop Reparatrice Appar Mot 1978;64:59-70.

5. Kamath J, Jayasheelan N, Mathews R. Compressive neuropathy of the palmar cutaneous branch of the median nerve after a malunited fracture of the distal radius. J Hand Surg Eur Vol 2016;41:231-2.

6. Carroll RE, Green DP. The significance of the palmar cutaneous nerve at the wrist. Clin Orthop Relat Res 1972;83:24-8.

7. Louis DS, Greene TL, Noellert RC. Complications of carpal tunnel surgery. J Neurosurg 1985;62:352-6.

8. Ilyas AM. Surgical approaches to the distal radius. Hand (N Y) 2011;6:8-17.

9. An H, Park S, Kim HD. The influence of stimulation site on the conduction study of palmar cutaneous branch of median nerve. J Korean Acad Rehabil Med 2002;26:46-9.

10. Imai T, Wada T, Matsumoto H. Entrapment neuropathy of the palmar cutaneous branch of the median nerve in carpal tunnel syndrome. Clin Neurophysiol 2004;115:2514-7.

11. Tagliafico A, Pugliese F, Bianchi S, Bodner G, Padua L, Rubino M, et al. High-resolution sonography of the palmar cutaneous branch of the median nerve. AJR Am J Roentgenol 2008;191:107-14.

12. Chang KV, Mezian K, Nanka O, Wu WT, Lou YM, Wang JC, et al. Ultrasound imaging for the cutaneous nerves of the extremities and relevant entrapment syn- 
dromes: from anatomy to clinical implications. J Clin Med 2018;7:E457.

13. McCann PA, Clarke D, Amirfeyz R, Bhatia R. The cadaveric anatomy of the distal radius: implications for the use of volar plates. Ann R Coll Surg Engl 2012;94:116-20.

14. Conti Mica MA, Bindra R, Moran SL. Anatomic considerations when performing the modified Henry approach for exposure of distal radius fractures. J Orthop 2016;14:104-7.

15. Hobbs RA, Magnussen PA, Tonkin MA. Palmar cutaneous branch of the median nerve. J Hand Surg Am 1990;15:38-43.

16. Gaspar MP, Sessions BA, Dudoussat BS, Kane PM. Single-incision carpal tunnel release and distal radius open reduction and internal fixation: a cadaveric study. J Wrist Surg 2016;5:241-6.

17. Jones C, Beredjiklian P, Matzon JL, Kim N, Lutsky K.
Incidence of an anomalous course of the palmar cutaneous branch of the median nerve during volar plate fixation of distal radius fractures. J Hand Surg Am 2016;41:841-4.

18. Berglund LM, Messer TM. Complications of volar plate fixation for managing distal radius fractures. J Am Acad Orthop Surg 2009;17:369-77.

19. Smith J, Wisniewski SJ, Finnoff JT, Payne JM. Sonographically guided carpal tunnel injections: the ulnar approach. J Ultrasound Med 2008;27:1485-90.

20. Kim DH, Jang JE, Park BK. Anatomical basis of ulnar approach in carpal tunnel injection. Pain Physician 2013;16:E191-8.

21. Cartwright MS, Walker FO. Neuromuscular ultrasound in common entrapment neuropathies. Muscle Nerve 2013;48:696-704.

22. Roche SJ, Ng CY. Absent palmar cutaneous branch of median nerve. J Hand Microsurg 2014;6:47-8. 